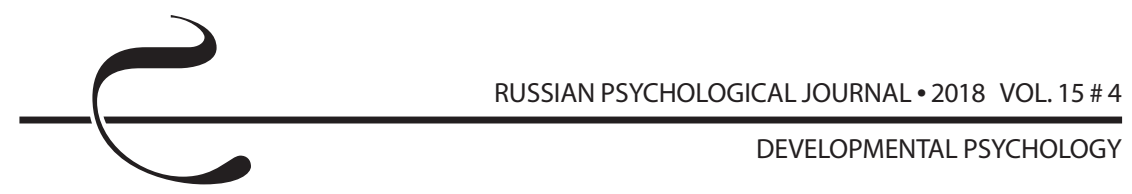

УДК 159.922.736.3

DOI: $\underline{10.21702 / r p j .2018 .4 .5}$

\title{
Возможности развития регуляторных функций в игровой деятельности: теоретический обзор
}

\author{
Александр Н. Веракса“, Дарья А. Бухаленкова, Вера А. Якупова \\ Московский государственный университет имени М. В. Ломоносова, г. Москва, \\ Российская Федерация \\ *E-mail: veraksa@yandex.ru
}

\begin{abstract}
Аннотация
Введение. Авторами Аоказывается актуальность рассмотрения потенциала игровой Аеятельности Аля развития различных высших психических функций в АОшкольном возрасте. Подчеркивается особое значение игры как символического пространства, через которое ребенок познает окружающий мир и овладевает своими психическими процессами.
\end{abstract}

Теоретическое обоснование. В разделе описываются компоненты регуляторных функций (рабочая память, сАерживающий контроль и когнитивная гибкость), и показано, как они задействованы в игровой деятельности.

Результаты. РазАел включает описание корреляционных исслеАований связи саморегуляции и игры, а также фрормирующих экспериментов, в которых игровая Аеятельность использовалась Аля развития регуляторных функций. Корреляционные исследования показывают, что свободная игра со сверстниками имеет сложную структуру и включает разные аспекты (принятие роли, игровое замещение предметов и Аействий, взаимолействие со сверстниками), которые по-разному связаны с показателями развития регуляторных функций у Аошкольников. ОтАельно описываются исслеАования, показывающие наличие связи использования Аетьми воображения в игровой ситуации и развития регу^яторных функций. Результаты фрормирующих экспериментов показывают, что А^я осуществления игровой Аеятельности необхолим определенный уровень развития регуляторных функций, при этом она является механизмом их развития. ОбсужАение результатов. Авторы рассматривают ограничения проанализированных исследований. Наиболее значимое ограничение связано с тем, что в большинстве рассмотренных исследований изучение игровой деятельности ограничивается анализом только одного из ее аспектов (игровое замещение, принятие роли, воображаемая ситуация). Проведенный обзор показывает необходимость вылеления целого ряда параметров и проведения Алительного наблюАения Аля описания игровой Аеятельности кажАого ребенка. Авторы отмечают сложность организации развития регуляторных функций в рамках формирующих исследований с использованием игровой Аеятельности: важно, 
чтобы игровая Аеятельность не использовалась ^ишь как контекст эмоциональной вовлеченности ребенка, и чтобы выбранная символическая ситуация была близка интересам ребенка. Аелается вывод о перспективности Аанного направления исслелований.

\section{КАючевые слова}

Аошкольный возраст, игра, регуляторные функции, рабочая память, когнитивная гибкость, слерживающий контроль, саморегуляция, воображение, игровое замещение, символические средства

\section{Основные положения}

- такие показатели игры, как принятие роли, игровое замещение и взаимолействие со сверстниками, неразрывно связаны с развитием регуляторных фрункций

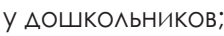

Аля осуществления игровой Аеятельности необхолим опреАеленный уровень развития регуляторных функций, олнако она также является механизмом их развития;

- воображаемая, символическая ситуация, эмоциональность которой залается присутствием воображаемого персонажа, позволяет выйти ребенку на новый уровень развития регуляторных функций, который неАоступен ему в реальной ситуации;

- налеление привычных объектов окружающей среды игровыми контекстами карАинально меняет произвольное поведение ребенка: процесс целеполагания носит ярко выраженный творческий характер, что требует от ребенка Аругого уровня когнитивной регуляции своей Аеятельности.

\section{Для цитирования}

Веракса А. Н., Бухаленкова Д. А., Якупова В. А. Возможности развития регуляторных функций в игровой деятельности: теоретический обзор // Российский психологический журнал. 2018. T. 15, № 4. C. 97-112. DOI: 10.21702/rpj.2018.4.5

Материалы статьи получены 13.06.2018 


\title{
Development of Executive Functions Through Play Activities: A Theoretical Overview
}

\author{
Aleksandr N. Veraksa*, Daria A. Bukhalenkova, Vera A. Yakupova \\ Lomonosov Moscow State University, Moscow, Russian Federation \\ *Corresponding author. E-mail: veraksa@yandex.ru
}

\begin{abstract}
Introduction. Play is a symbolic space through which children cognize the world around them and master their mental processes. This study considers the potential of play activity for the development of various higher mental functions in preschool-age children.

Theoretical Basis. This section describes working memory, inhibition, and cognitive flexibility as components of executive functions and demonstrates how they are involved in play activity.
\end{abstract}

Results. This section summarizes: a) correlation studies on the relationship between self-regulation and play; and b) formative experiments that used play activity for the development of executive functions. Correlation studies showed that free play with peers has a complex structure and includes various aspects (role-taking, play substitution of objects and actions, and interaction with peers), which are differently related to the development of executive functions in preschool children. Attention is also given to studies that establish the relationship between children's imagination in a play situation and the development of executive functions. Formative experiments indicated that a certain level of the development of executive functions is important for the implementation of play activities. At the same time, play activity is a mechanism for the development of executive functions.

Discussion. The authors concentrate on limitations of the analyzed studies. The major drawback they identified is that previous studies of play activity examined its certain components in isolation (object substitution, role-taking, and imaginary situation). This review calls for further research that would identify a number of parameters and undertake a long-term observation to describe play activity of every individual child. Play activity is not simply a context for children's emotional involvement; it is also important for the symbolic situations of the play to be close to their interests. Thus, the authors establish the complexity of organizing the development of executive functions within formative experiments using play activity. The study emphasizes the great potential of this direction of research.

\section{Keywords}

preschool age, play, executive functions, working memory, cognitive flexibility, inhibition, self-regulation, imagination, object substitution, symbolic tools 


\section{Highlights}

- Role-taking ability, object substitution, and interaction with peers in play are inseparably linked to the development of executive functions in preschool-age children.

- A certain level of the development of executive functions is instrumental for the implementation of play activities. In turn, play activity is a mechanism for the development of executive functions.

- An imaginary symbolic situation, which is emotionally loaded by the presence of an imaginary character, enables a child to achieve a new level of the development of executive functions.

Attributing play contexts to common objects of the environment radically changes a child's arbitrary behavior. Goal-setting in play is a creative process that requires a different level of children's cognitive regulation of their activity.

\section{For citation}

Veraksa A. N., Bukhalenkova D. A., Yakupova V. A. Development of Executive Functions Through Play Activities: A Theoretical Overview. Rossiiskii psikhologicheskii zhurnal-Russian Psychological Journal, 2018, V. 15, no. 4, pp. 97-112 (in Russian). DOI: 10.21702/rpj.2018.4.5

Original manuscript received 13.06.2018

\section{Введение}

Игра представляет собой символическое пространство, которое выстраивается ребенком и служит познанию сложного окружающего мира. Выступая в качестве ведущей деятельности, согласно А. Н. Леонтьеву [1], игровая форма познания предоставляет ребенку преимущества в сравнении, например, со знаковой формой.

В психологической науке накоплен огромный массив данных, который показывает потенциал игровой деятельности для развития мышления [например, 2, 3], воображения [3], памяти [например, 4] и других психических функций [5]. Так, в игровой ситуации дети 4-6 лет способны решать силлогизмы, что вызывает у них трудности в ситуации реальной. Если спросить ребенка о том, какие будут последствия игры в футбол мамы, то большинство детей ответит, что мама не играет в футбол. Если же спросить, что будет, если в футбол будет играть игрушечный дракон, то дети дадут верный ответ, согласно которому дракон испачкается, устанет и т. д. [2]. Игровая деятельность является более эффективной для организации запоминания дошкольниками. Как показали исследования 3. М. Истоминой [4], успешность запоминания в игровой деятельности превышает в 3-7 раз успешность запоминания в лабораторной ситуации, что подчеркивает значимость связи мотива запоминания с игровым мотивом. Проведенный метаанализ более 40 исследований показал, что уровень 
развития игровой деятельности положительно связан с развитием дивергентного мышления и креативности ребенка. В лонгитюдном исследовании С. Расс и коллег [5] на протяжении четырех лет проводилось наблюдение за игрой детей. Оказалось, что дети с высоким уровнем развития фантазии в игре могли предложить больше способов совладания со стрессовой ситуацией.

Эти и многие другие исследования подчеркивают важность игрового пространства как особого пространства, в котором, по мнению Л. С. Выготского [6], ребенок действует с объектом одновременно и как с реальным и как с воображаемым, что способствует развитию произвольной регуляции.

\section{Теоретическое обоснование}

Регуляторные функции рассматриваются как высшие процессы, которые позволяют контролировать поведение, делая его более адаптивным и целенаправленным. Среди наиболее важных когнитивных процессов, которые включены в регуляторные функции, выделяют рабочую память, сдерживающий контроль и когнитивную гибкость [7].

Одним из основных компонентов игровой деятельности является принятие ребенком различных ролей. Принимая на себя роль, дошкольник, с одной стороны, следует тем правилам и предписаниям, которые несет роль, а с другой - в любой момент времени может быть осуществлен выход из роли, т. е. смена игровой ситуации на ситуацию реальную. В игровой деятельности задействованы все компоненты регуляторных функций: когнитивная гибкость (переключение с одной роли на другую, с игровой деятельности на деятельность реальную), сдерживающий контроль (использование игрового замещения, т. е. сдерживание отношения к игровой ситуации как к ситуации реальной и действие по правилам игры), рабочая память (предполагающая удержание игровых правил и др.). Кроме того, в игре осуществляется взаимодействие ребенка со сверстниками, в результате чего дети начинают учитывать желания и действия другого ребенка, контролировать свое поведение и реализовывать совместные планы. В этой связи игровая деятельность традиционно рассматривается как важная для развития произвольности ребенка [8].

В связи с этим, целью данного обзора было проанализировать исследования, направленные на изучение взаимосвязи регуляторных функций и игровой деятельности дошкольников за последние 15 лет.

\section{Результаты \\ Корреляционные исследования взаимосвязи РФ и игры в дошкольном возрасте}

В ряде корреляционных исследований было показано наличие связи уровня развития игры и саморегуляции у дошкольников $[9,10,11]$. 
Так, в исследовании П. Слот и коллег [9] развитие игровой деятельности сопоставлялось с саморегуляцией детей. При этом авторы выделили когнитивный, поведенческий и эмоциональный компоненты саморегуляции, которые проявлялись в игре. Когнитивная саморегуляция проявлялась в планировании, эмоциональная - в осведомленности о собственных эмоциях, поведенческая в умении следовать правилам и установленным договоренностям. Развитие игровой деятельности оценивалось по таким показателям, как принятие роли, игровое замещение предметов и действий, взаимодействие со сверстниками. Были обнаружены значимые взаимосвязи между уровнем развития игровой деятельности и уровнем когнитивной саморегуляции, демонстрируемым в ней. На наш взгляд, данное исследование ставит вопрос о внутренней связи между развитием игры и саморегуляции: такие показатели, как принятие роли, игровое замещение и взаимодействие со сверстниками, неразрывно связаны с регуляцией собственного поведения ребенком.

В исследовании А. Ивренди [12] проводилось сопоставление развития игровой деятельности и развития саморегуляции у 148 детей в возрасте 5 лет. В рамках измерений игра была разделена на игру низкого уровня (одиночную, параллельную), интерактивную игру (предполагающую глазной контакт с партнером, простое социальное взаимодействие) и компетентную игру, предполагающую полноценное взаимодействие. Результаты показали устойчивую связь между уровнем развития игры и уровнем регуляторных функций.

Исследование Дж. Фантуззо и коллег [13] также было направлено на изучение взаимосвязи саморегуляции у детей в возрасте от 3 до 6 лет и особенностей их взаимодействия со сверстниками в игре. Дети, демонстрировавшие более развитые навыки общения со сверстниками во время свободной игры (способные эффективно разрешать возникающие противоречия, не склонные к агрессивному поведению), обладали также более высоким уровнем развития эмоциональной регуляции. Исследование также показало, что дети, которые свободно вступали в игру со сверстниками, проявляли креативность в развитии игровых сюжетов и больше инициативности и автономии во время учебных занятий.

В работе С. Элиас и Л. Берк [11] исследователи наблюдали за детьми дважды: вначале были изучены навыки саморегуляции детей и развитие игровой деятельности, а спустя полгода повторно осуществлялось наблюдение за саморегуляцией детей. Оказалось, что присутствует положительная взаимосвязь развития навыков саморегуляции детей (которые проявлялись, например, в умении убирать игрушки) с уровнем развития игры. При этом данная взаимосвязь прослеживалась относительно второго замера саморегуляции, что говорит, по мнению авторов, о прогностичном характере показателя развития игры. Обнаруженная закономерность была сильнее для 


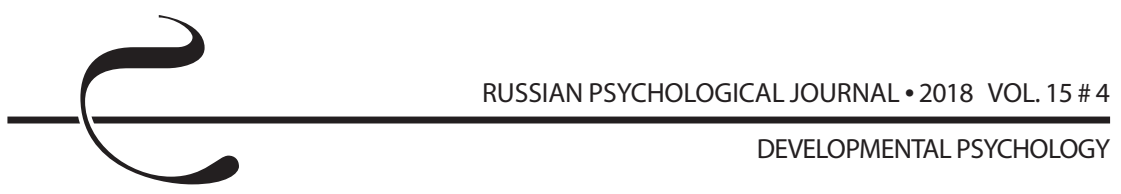

высокого уровня развития игры-драматизации, а не самого факта наличия или отсутствия игры у детей.

Таким образом, результаты нескольких корреляционных исследований показали устойчивую связь между уровнем взаимодействия детей в игре и уровнем регуляторных функций $[12,13,14]$. Вместе с тем, в исследовании R. Kelly и коллег [15] связь между выполнением детьми методик на диагностику регуляторных функций и наблюдением за свободной игрой не была выявлена. Существующие исследования показывают, что игра имеет сложную структуру и включает разные аспекты (игровое замещение предметов и действий, взаимодействие со сверстниками, принятие роли), которые по-разному связаны с показателями развития регуляторных функций у дошкольников. При этом в ряде исследований показана связь между использованием воображения в игровой ситуации и развитием регуляторных функций $[16,17,18,19,20]$.

\section{Исследования взаимосвязи использования детьми воображения в игре и регуляторных функций в дошкольном возрасте}

Установление границ между реальностью и воображаемой ситуацией, начиная с исследований Л. С. Выготского, выступало основой для появления подлинной игровой деятельности. И. Сигель [21] был использован термин «психологическое дистанцирование», под которым понималось поведение или событие, которое в когнитивном плане отделяло ребенка от его непосредственного окружения, что приводило к развитию репрезентации. В данной традиции развитие игровой деятельности тесно связывалось со становлением двойного кодирования.

В наших исследованиях было показано, что использование символического пространства в решении познавательных задач выступает как зона ближайшего развития для детей, которые испытывают сложности в освоении знаковых средств [22]. При этом движение в зоне ближайшего развития, по выражению А. Лиллард [23], происходит фактически самостоятельно, без активного участия взрослого.

В классической экспериментальной ситуации отложенного поощрения, когда дети были вынуждены длительное время сидеть непосредственно перед привлекательным и съедобным стимулом (зефирки) для того, чтобы впоследствии получить от взрослого его большее количество, более успешными были дети, которые смогли в воображаемой ситуации представить зефирки как некоторые абстрактные объекты (например, облака) [16].

Результаты исследования Дж. Перуччи и коллег [24] показали, что мысли детей, связанные с работой их фантазии (например, наличие воображаемых друзей, вовлечение в воображаемую игру или другие проявления воображаемого мира), значимо связаны с развитием когнитивной гибкости 
и сдерживающего контроля. Однако игровые действия, такие как действия с воображаемой игрушкой или действия в процессе воображаемой игры, были негативно связаны с этими показателями регуляторных функций. Авторы интерпретируют полученные данные, акцентируя важность когнитивных аспектов фантазии для развития саморегуляции, но не поведенческих ее проявлений.

В работе С. Карлсон и коллег [18] была показана устойчивая положительная связь регуляторных функций и выполнения воображаемых действий детьми в возрасте четырех лет. Полученные результаты подтвердили выводы предыдущих исследований, полученных на более развернутой выборке [17]. С точки зрения авторов, данное обстоятельство иллюстрирует необходимость определенного уровня развития регуляторных функций для осуществления воображаемых действий и впоследствии развертывания игровой деятельности. Безусловно, определенный уровень развития регуляции для выполнения игровых действий необходим. Однако именно развитие игры является мощным мотивационным фактором развития регуляции.

В работе Р. Келли и С. Хаммонд [15] была получена значимая связь сдерживающего контроля со структурированной символической игрой на относительно небольшой выборке в 20 детей в возрасте 4-6 лет. Чем выше был уровень развития игры (использование объектов-заместителей, воображение отсутствующих объектов, приписывание объектам воображаемых свойств, принятие роли ребенком), тем выше был уровень развития указанного показателя регуляторных функций. Небольшая выборка детей, к сожалению, не позволяет сделать уверенных выводов об обнаруженной взаимосвязи, однако задает направление исследований, представляющее несомненный интерес.

\section{Использование игровой деятельности для влияния на развитие регуляторных функций}

Перед описанием исследований, в которых были предприняты попытки развития регуляторных функций с помощью вовлечения детей в игровую деятельность, остановимся на исследовании, в котором воображаемая ситуация была использована для активизации потенциала развития регуляторных функций детей. Подтверждение продуктивности данного подхода можно найти в относительно недавнем исследовании Р. Е. Уайт и коллег [19], посвященном изучению влияния роли, которую берет на себя ребенок, на успешность выполнения заданий, направленных на изучение регуляторных функций. Задания предъявлялись в трех вариантах: традиционном, в ситуации, когда ребенок представлял другого ребенка, который выполнял задание («Как Джон считает, куда относится эта карточка?»), и когда ребенок выполнял задание от лица вымышленного персонажа («Теперь ты Бэтмен! Куда Бэтмен 


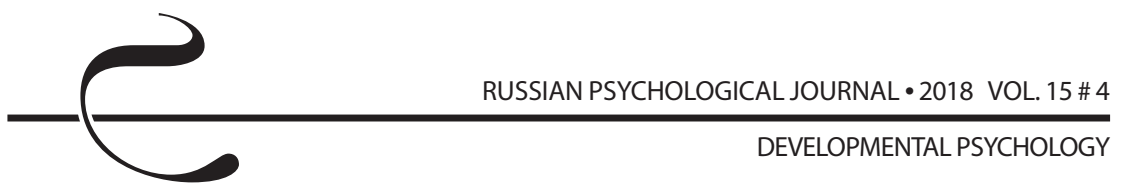

положит эту карточку?»). Результаты исследования показали, что выполнение заданий от третьего лица значимо повышает правильность их решения. При этом выполнение детьми заданий в ситуации с вымышленным персонажем было успешнее в сравнении с ситуацией, когда речь шла о другом ребенке. Авторы интерпретируют полученные результаты как показывающие важность психологического дистанцирования детей и его связь с демонстрируемым уровнем развития саморегуляции.

Возвращаясь к описанному выше исследованию С. Карлсон и коллег [18], полученные данные говорят в пользу того, что игровая деятельность не только нуждается в определенном уровне развития регуляторных функций, но и является механизмом их развития. Нам представляется важным то обстоятельство, что воображаемая символическая ситуация, эмоциональность которой задается присутствием воображаемого персонажа, позволяет ребенку выйти на новый уровень развития регуляторных функций, который недоступен ему в реальной ситуации.

В исследовании Р. Тибодо и коллег [20] приняли участие 110 детей в возрасте 3-5 лет. В рамках претеста изучалось развитие трех основных компонентов регуляторных функций у детей. Затем дети были разделены на три группы: группу, в которой осуществлялась воображаемая игра, группу, в которой осуществлялась игра без воображения, и контрольную группу, в которой распорядок дня и активности не менялись. Воображаемая игра представляла собой разворачивание фантастических сюжетов, которые задавал взрослый (например, прогулка на Луне), а развивали их дети. В группе, где игры не были связаны с воображением, детям предлагались игры с мячом, раскраски, песни и другие активности, которые целенаправленно не задействовали регуляторные функции. Эксперимент длился на протяжении 5 недель, в рамках которых с детьми занимались в мини-группах по 5-6 человек ежедневно в течение 15 минут. По окончании эксперимента был проведен посттест, направленный на измерение развития регуляторных функций. Результаты показали, что дети, которые были вовлечены в воображаемую игру, значимо повысили уровень рабочей памяти и когнитивной гибкости в сравнении с детьми из других групп. Авторы утверждают, «что действие переключения между реальностью и воображением... это то, что ведет за собой достижения в развитии регуляторных функций, подобно когнитивным достижениям, наблюдаемым в случае переключения между языками в билингвальном окружении» [20, с. 135].

Заметим, что фактор времени, которое уделяется развитию игровой деятельности, является важным при планировании интервенции. Так, в исследовании Л. Ку и коллег [25] дошкольников обучали игре-драматизации на протяжении четырех встреч. Однако никаких эффектов на развитие регуляторных функций это не оказало. В то же время, хотя длительный опыт использования 
образовательных программ для детей дошкольного возраста, в которых игровой деятельности и развитию регуляторных функций уделяется большое внимание, и показывает позитивные изменения в развитии саморегуляции, структура программ не позволяет выделить вклад фактора игровой деятельности среди других факторов [26].

\section{Обсуждение результатов}

В описанных нами экспериментальных исследованиях результаты говорят о положительной взаимосвязи регуляторных функций и игры у дошкольников. При этом данная связь прослеживается не только на уровне корреляционных исследований, но и оказывается ярко выраженной в исследованиях формирующих.

Представленные исследования не лишены ограничений. Во-первых, как правило, в них речь идет об относительно небольших выборках детей, относящихся к различным возрастным группам в диапазоне от 3 до 6 лет, что затрудняет сопоставление полученных норм и прослеживание закономерностей на протяжении 2-3 лет.

Во-вторых, в рассмотренных исследованиях в большинстве случаев изучение игровой деятельности ограничивается анализом одного из ее аспектов (игровое замещение, принятие роли, воображаемая ситуация). К тому же используемые в исследованиях методические приемы помогают констатировать наличие или отсутствие выполнения воображаемого действия, принятие роли и т.д. в экспериментально заданной ситуации. Однако осуществление подобных процедур в лабораторных условиях, с точки зрения теории игры в рамках культурно-исторической психологии и теории деятельности, не позволяет говорить о подлинном разворачивании полноценной игровой деятельности, предполагающей соответствующую мотивацию, взаимодействие со сверстниками и т. д. [27]. Наблюдения за игровой деятельностью в группе, описанные в рамках анализируемых исследований, также не затрагивают все существенные ее компоненты, а сводятся или к наличию, или отсутствию игрового взаимодействия. Очевидно, что для описания игровой деятельности каждого конкретного ребенка требуется выделение целого ряда параметров и, что наиболее важно, длительное наблюдение за его игрой. Данные требования к оценке игры у дошкольников являются причиной того, что при высокой актуальности рассматриваемой проблемы ее решению посвящено не так уж много работ.

Говоря о формирующих исследованиях, необходимо также отметить сложность организации развития регуляторных функций в рамках игровой деятельности. Важно, чтобы, с одной стороны, игровая деятельность не использовалась лишь как контекст эмоциональной вовлеченности ребенка, а с другой - чтобы подобранная символическая ситуация была близка интересам ребенка. Как показали наши исследования, подобрать символ, который 


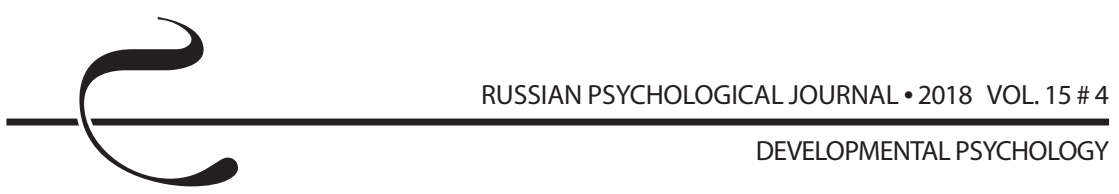

оказал бы развивающий эффект на формирование, например, двигательного навыка, представляет достаточно сложную специальную задачу [28].

Полученные авторами результаты мы рассматриваем как следствие сложной организации игровой деятельности. Дело в том, что мир взрослых для маленького человека выступает в своей тотальной неопределенности. Как было показано в целом ряде исследований, оказавшись в ситуации неопределенности, дети начинают применять символические средства $[29,30]$. Их основная особенность, в отличие от знаковых средств, заключается в двойной предметности. Игровая ситуация характеризуется тем, что окружающие ребенка объекты вовлекаются в игру только в том случае, если они, помимо основных значений, характерных для повседневной жизни, приобретают еще и игровые контексты. Наделение объектов игровыми контекстами кардинально меняет произвольное поведение ребенка. Прежде всего, по-другому протекает процесс целеполагания. Цель игрового действия определяется не непосредственными желаниями дошкольника, а другими детерминантами: особенностями разворачиваемого на данный момент игрового сюжета и возможностью использования объектов, которые находятся вокруг ребенка в адекватных игровых контекстах. Важно отметить, что объект вовлекается в игру только в том случае, если он приобретает новый игровой контекст. В процессе целеполагания ребенок должен не только удерживать уровень развития сюжета и осуществлять постоянное «примеривание» контекстов к окружающим объектам, но и осуществлять поиск новых вариантов движения игровой деятельности, задающих возможности новых контекстов. Другими словами, процесс целеполагания носит ярко выраженный творческий характер, что требует от ребенка другого уровня регуляции. Кроме того, вводя новые предметы-заместители, ребенок должен распределять внимание между старыми объектами (уже имеющими игровые контексты) и анализировать возможности вовлечения новых объектов в игру. Удержание приобретенных контекстов требует развития рабочей памяти детей.

Очевидно, что относительно небольшое количество исследований, рассмотренных в предложенном обзоре, говорит как о трудностях, так и о перспективности данного направления исследований. Популярность подхода Л. С. Выготского к образованию применительно к дошкольному детству [31] позволяет ожидать появления новых работ, направленных на раскрытие развивающего потенциала игровой деятельности.

\section{Благодарности}

Работа выполнена при поддержке гранта РФФИ № 18-013-01057.

\section{Acknowledgments}

Supported by the Russian Foundation for Basic Research, grant 18-013-01057. 


\section{Литература}

1. Леонтьев А. Н. Психологические основы дошкольной игры // Психологическая наука и образование. 1996. № 3. С. 19-31.

2. KuczajS. A. Factors influencing children's hypothetical reference // Journal of Child Language. 1981. Vol. 8, Issue 1. P. 131-137. DOI: $10.1017 /$ S0305000900003056

3. Fisher E. P. The impact of play on development: A meta-analysis // Play \& Culture. 1992.5 (2). P. 159-181.

4. Истомина 3. М. Развитие памяти. М.: Психология, 1977. 120 с.

5. Russ S. W., Robins A. L., Christiano B. A. Pretend Play: Longitudinal Prediction of Creativity and Affect in Fantasy in Children // Creativity Research Journal. 1999. Vol. 12, Issue 2. P. 129-139. DOI: $10.1207 /$ s15326934crj1202 5

6. Выготский Л. С. Игра и ее роль в психическом развитии ребенка // Психология развития ребенка. М.: Смысл, Эксмо, 2004. С. 200-223.

7. Miyake A., Friedman N. P., Emerson M. J., Witzki A. H., Howerter A., Wager T. D. The Unity and Diversity of Executive Functions and Their Contributions to Complex "Frontal Lobe" Tasks: A Latent Variable Analysis // Cognitive Psychology. 2000. Vol. 41, Issue 1. P. 49-100. DOI: 10.1006/cogp.1999.0734

8. Эльконин Д. Б. Психология игры. М.: Педагогика, 1976. 304 с.

9. Slot P. L., Mulder H., Verhagen J., Leseman P. P. M. Preschoolers' cognitive and emotional self-regulation in pretend play: Relations with executive functions and quality of play // Infant and Child Development. 2017. Vol. 26, Issue 6. e2038. DOI: $10.1002 / \mathrm{icd} .2038$

10. Zyga O. The Act of Pretending: Play, Executive Function, and Theory of Mind in Early Childhood. 2016. 76 p. URL: http://rave.ohiolink.edu/etdc/ view?acc num=case1467391080 (Accessed 30 October 2018).

11. Elias C. L., Berk L. E. Self-regulation in young children: Is there a role for sociodramatic play? // Early Childhood Research Quarterly. 2002. Vol. 17, Issue 2. P. 216-238. DOI: 10.1016/S0885-2006(02)00146-1

12. Ivrendi $A$. Choice-driven peer play, self-regulation and number sense // European Early Childhood Education Research Journal. 2016. Vol. 24, Issue 6. P. 895-906. DOI: $10.1080 / 1350293 X .2016 .1239325$

13. Fantuzzo J., Sekino Y., Cohen H. L. An examination of the contributions of interactive peer play to salient classroom competencies for urban head start children // Psychology in the Schools. 2004. Vol. 41, Issue 3. P. 323-336. DOI: $10.1002 /$ pits.10162

14. Mikami A. Y. The Importance of Friendship for Youth with Attention-Deficit/ Hyperactivity Disorder // Clinical Child and Family Psychology Review. 2010. Vol. 13, Issue 2. P. 181-198. DOI: 10.1007/s10567-010-0067-y

15. Kelly R., Dissanayake C., Ihsen E., Hammond S. The Relationship between Symbolic Play and Executive Function in Young Children // Australasian 
Journal of Early Childhood. 2011. Vol. 36, № 2. P. 21-28.

16. Mischel W., Baker N. Cognitive appraisals and transformations in delay behavior // Journal of Personality and Social Psychology. 1975. Vol. 31. P. 254-261.

17. Vieillevoye S., Nader-Grosbois N. Self-Regulation during Pretend Play in Children with Intellectual Disability and in Normally Developing Children // Research in Developmental Disabilities. 2008. Vol. 29, № 3. P. 256-272.

18. Carlson S. M., White R. E., Davis-Unger A. C. Evidence for a relation between executive function and pretense representation in preschool children // Cognitive Development. 2014.Vol. 29. P. 1-16. DOI:10.1016/j.cogdev.2013.09.001

19. White R. E., Carlson S. M. What would Batman do? Self-distancing improves executive function in young children // Developmental Science. 2016. Vol. 19, Issue 3. P. 419-426. DOI: 10.1111/desc.12314

20. Thibodeau R. B., Gilpin A. T., Brown M. M., Meyer B. A. The effects of fantastical pretend-play on the development of executive functions: An intervention study // Journal of Experimental Child Psychology. 2016. Vol. 145. P. 120-138. DOI: $10.1016 /$ j.jecp.2016.01.001

21. Sigel I. E. The distancing hypothesis: A causal hypothesis for the acquisition of representational thought // Miami Symposium on the Prediction of Behavior, 1968: Effects of Early Experience / M. R. Jones (Ed.). Coral Gables, FL: University of Miami Press, 1970. P. 99-118.

22. Веракса А. Н., Горовая А. Е., Кисель А. В. Возможности использования знаковых и символических средств в обучении дошкольников (на примере освоения феномена радуги) // Психологическая наука и образование psyedu.ru. 2014. T. 6, № 2. C. 19-34. URL: http://psyedu.ru/journal/2014/2/ Veraksa Gorovaya Kisel.phtml (дата обращения: 30.10.2018).

23. Lillard A. S., Lerner M. D., Hopkins E. J., Dore R. A., Smith E. D., Palmquist C. M. The impact of pretend play on children's development: A review of the evidence // Psychological Bulletin. 2013. Vol. 139 (1). P. 1-34. DOI: $10.1037 / a 0029321$

24. Pierucci J. M., O'Brien C. T., Mclnnis M. A., Gilpin A. T., Barber A. B. Fantasy orientation constructs and related executive function development in preschool: Developmental benefits to executive functions by being a fantasy-oriented child // International Journal of Behavioral Development. 2014. Vol. 38, Issue 1. P. 62-69. DOI: $10.1177 / 0165025413508512$

25. Qu L., Shen P., Chee Y. Y., Chen L. Teachers' Theory-of-mind Coaching and Children's Executive Function Predict the Training Effect of Sociodramatic Play on Children's Theory of Mind // Social Development. 2015. Vol. 24, Issue 4. P. 716-733. DOI: $10.1111 /$ sode. 12116

26. Shaheen S. How Child's Play Impacts Executive Function - Related Behaviors // Applied Neuropsychology: Child. 2014. Vol. 3, Issue 3. P. 182-187. DOI: $\underline{10.1080 / 21622965.2013 .839612}$ 
27. Смирнова Е. О., Веракса А. Н., Бухаленкова Д. А., Рябкова И. А. Связь игровой деятельности дошкольников с показателями познавательного развития // Культурно-историческая психология. 2018. Т. 14, № 1. С. 4-14. DOI: $10.17759 /$ chp.2018140101

28. Veraksa A. N., Gorovaya A. E., Leonov S. V., Pashenko A. K., Fedorov V. V. The Possibility of Using Sign and Symbolic Tools in the Development of Motor Skills by Beginning Soccer Players // Psychology in Russia: State of the Art. 2012. Vol. 5. P. 473-497. DOI: $10.11621 /$ pir.2012.0030

29. Веракса А. Н. Символическое опосредствование в познавательной деятельности: Монография. М.: Изд-во МГУ, 2010. 151 с.

30. Субботский Е. В. Строящееся сознание. М.: Смысл, 2007. 423 с.

31. Vygotsky's Theory in Early Childhood Education and Research: Russian and Western values / N. Veraksa, S. Sheridan (Eds.). London; New York: Routledge, Taylor \& Francis Group, 2018. 219 p.

\section{References}

1. Leont'ev A. N. Psychological foundations of preschool play. Psikhologicheskaya nauka i obrazovanie - Psychological Science and Education, 1996, no. 3, pp. 19-31 (in Russian).

2. Kuczaj S. A. Factors influencing children's hypothetical reference. Journal of Child Language, 1981, V. 8, Issue 1, pp. 131-137. DOI: $10.1017 /$ S0305000900003056

3. Fisher E. P. The impact of play on development: A meta-analysis. Play \& Culture, 1992, 5 (2), pp. 159-181.

4. Istomina Z. M. Razvitie pamyati [Development of memory]. Moscow, Psikhologiya Publ., 1977. 120 p.

5. Russ S. W., Robins A. L., Christiano B. A. Pretend play: Longitudinal prediction of creativity and affect in fantasy in children. Creativity Research Journal, 1999, V. 12, Issue 2, pp. 129-139. DOI: 10.1207/s15326934crj1202 5

6. Vygotskii L. S. Play and its role in the mental development of the child. In: Psikhologiya razvitiya rebenka [Psychology of child development]. Moscow, Smysl, Eksmo Publ., 2004, pp. 200-223.

7. Miyake A., Friedman N. P., Emerson M. J., Witzki A. H., Howerter A., WagerT. D. The unity and diversity of executive functions and their contributions to complex "frontal lobe" tasks: A latent variable analysis. Cognitive Psychology, 2000, V. 41, Issue 1, pp. 49-100. DOI: 10.1006/cogp.1999.0734

8. El'konin D. B. Psikhologiya igry [Psychology of play]. Moscow, Pedagogika Publ., 1976. 304 p.

9. Slot P. L., Mulder H., Verhagen J., Leseman P. P. M. Preschoolers' cognitive and emotional self-regulation in pretend play: Relations with executive 
functions and quality of play. Infant and Child Development, 2017, V. 26, Issue 6, e2038. DOI: 10.1002/icd.2038

10. Zyga O. The act of pretending: Play, executive function, and theory of mind in early childhood, 2016. 76 p. URL: http://rave.ohiolink.edu/etdc/view?acc num=case1467391080 (Accessed 30 October 2018).

11. Elias C. L., Berk L. E. Self-regulation in young children: Is there a role for sociodramatic play? Early Childhood Research Quarterly, 2002, V. 17, Issue 2, pp. 216-238. DOI: 10.1016/S0885-2006(02)00146-1

12. Ivrendi A. Choice-driven peer play, self-regulation and number sense. European Early Childhood Education Research Journal, 2016, V. 24, Issue 6, pp. 895-906. DOI: 10.1080/1350293X.2016.1239325

13. Fantuzzo J., Sekino Y., Cohen H. L. An examination of the contributions of interactive peer play to salient classroom competencies for urban head start children. Psychology in the Schools, 2004, V. 41, Issue 3, pp. 323-336. DOI: $10.1002 /$ pits.10162

14. Mikami A. Y.The importance of friendship for youth with attention-deficit/ hyperactivity disorder. Clinical Child and Family Psychology Review, 2010, V. 13, Issue 2, pp. 181-198. DOI: 10.1007/s10567-010-0067-y

15. Kelly R., Dissanayake C., Ihsen E., Hammond S. The relationship between symbolic play and executive function in young children. Australasian Journal of Early Childhood, 2011, V. 36, no. 2, pp. 21-28.

16. Mischel W., Baker N. Cognitive appraisals and transformations in delay behavior. Journal of Personality and Social Psychology, 1975, V. 31, pp. 254-261.

17. Vieillevoye S., Nader-Grosbois N. Self-Regulation during pretend play in children with intellectual disability and in normally developing children. Research in Developmental Disabilities, 2008, V. 29, no. 3, pp. 256-272.

18. Carlson S. M., White R. E., Davis-Unger A. C. Evidence for a relation between executive function and pretense representation in preschool children. Cognitive Development, 2014, V. 29, pp. 1-16. DOI: 10.1016/j.cogdev.2013.09.001

19. White R. E., Carlson S. M. What would Batman do? Self-distancing improves executive function in young children. Developmental Science, 2016, V. 19, Issue 3, pp. 419-426. DOI: 10.1111/desc.12314

20. Thibodeau R. B., Gilpin A. T., Brown M. M., Meyer B. A. The effects of fantastical pretend-play on the development of executive functions: An intervention study. Journal of Experimental Child Psychology, 2016, V. 145, pp. 120-138. DOI: $10.1016 /$ j.jecp.2016.01.001

21. Sigel I. E. The distancing hypothesis: A causal hypothesis for the acquisition of representational thought. In: M. R. Jones (ed.) Miami symposium on the prediction of behavior, 1968: Effects of early experience. Coral Gables, FL, University of Miami Press, 1970, pp. 99-118. 
22. Veraksa A. N., Gorovaya A. E., Kisel' A. V. The possibility of using iconic and symbolic tools in teaching (example of the acquisition of the rainbow phenomenon). Psychological Science and Education psyedu.ru, 2014, V. 6, no. 2, pp. 19-34 (in Russian). Available at: http://psyedu.ru/journal/2014/2/ Veraksa Gorovaya Kisel.phtml (Accessed 30 October 2018).

23. Lillard A. S., Lerner M. D., Hopkins E. J., Dore R. A., Smith E. D., Palmquist C. M. The impact of pretend play on children's development: A review of the evidence. Psychological Bulletin, 2013, V. 139 (1), pp. 1-34. DOI: $10.1037 /$ a0029321

24. Pierucci J. M., O’Brien C. T., Mclnnis M. A., Gilpin A. T., Barber A. B. Fantasy orientation constructs and related executive function development in preschool: Developmental benefits to executive functions by being a fantasyoriented child. International Journal of Behavioral Development, 2014, V. 38, Issue 1, pp. 62-69. DOI: 10.1177/0165025413508512

25. Qu L., Shen P., Chee Y. Y., Chen L. Teachers' theory-of-mind coaching and children's executive function predict the training effect of sociodramatic play on children's theory of mind. Social Development, 2015, V. 24, Issue 4, pp. 716-733. DOI: $10.1111 /$ sode. 12116

26. Shaheen S. How child's play impacts executive function - related behaviors. Applied Neuropsychology: Child, 2014, V. 3, Issue 3, pp. 182-187. DOI: $10.1080 / 21622965.2013 .839612$

27. Smirnova E. O., Veraksa A. N. Relationship between play activity and cognitive development in preschool children. Kul'turno-istoricheskaya psikhologiyaCultural-Historical Psychology, 2018, V. 14, no. 1, pp. 4-14 (in Russian). DOI: 10.17759/chp.2018140101

28. Veraksa A. N., Gorovaya A. E., Leonov S. V., Pashenko A. K., Fedorov V. V. The possibility of using sign and symbolic tools in the development of motor skills by beginning soccer players. Psychology in Russia: State of the Art, 2012, V. 5, pp. 473-497. DOI: 10.11621/pir.2012.0030

29. Veraksa A. N. Simvolicheskoe oposredstvovanie v poznavatel'noi deyatel'nosti [Symbolic mediation in cognitive activity]. Moscow, Moscow State University Publ., 2010. 151 p.

30. Subbotskii E. V. Stroyashcheesya soznanie [Consciousness under construction]. Moscow, Smysl Publ., 2007.423 p.

31. Veraksa N., Sheridan S. (eds.) Vygotsky's theory in early childhood education and research: Russian and Western values. London; New York: Routledge, Taylor \& Francis Group, 2018. 219 p. 